\title{
Late Embryogenesis Abundant (LEA) proteins confer water stress tolerance to
}

4 Czernik $M^{1 ; 2 ; 7}$., Fidanza $A^{1 ; 3 ; 7}$., Luongo FP $^{1 ; 4}$., Valbonetti $L^{5}$., Scapolo PA ${ }^{1}$., Patrizio $P^{6}$.,

\section{Loi $\mathbf{P}^{1^{*}}$}

6

$7 \quad{ }^{1}$ Faculty of Veterinary Medicine, University of Teramo, Teramo, Italy

$8 \quad{ }^{2}$ Department of Experimental Embryology, Institute of Genetics and Animal Breeding, Polish

9 Academy of Sciences, Jastrzebiec, Poland

$10{ }^{3}$ Current address: Centre for Regenerative Medicine, University of Edinburgh, Edinburgh, UK

$11{ }^{4}$ Current address: Center for Neurovirology, Department of Neuroscience, Lewis Katz School

12 of Medicine at Temple University, Philadelphia, PA 19140, USA

$13{ }^{5}$ Faculty of Biosciences and Technology for Food, Agriculture and Environment, University of

14 Teramo, 64100 Teramo, Italy.

$15{ }^{6}$ Yale Fertility Center, New Haven, CT 06511, USA.

$16{ }^{7}$ co-first authorship

$17 *$ Corresponding author

Corresponding author: Pasqualino Loi, Faculty of Veterinary Medicine, University of

Teramo, 64100 Teramo, Italy; phone number: +39 0861266 856; ploi@ unite.it,

23 Key words: Late Embryogenesis Abundant (LEA) proteins, xeroprotectants, mammalian 24 somatic cells, desiccation, 


\section{Abstract}

28 Late Embryogenesis Abundant (LEA) proteins are commonly found in organisms capable 29 of undergoing reversible dehydration - "anhydrobiosis". Here, we have produced three 30 LEA proteins: pTag-RAB17-GFP-N, Zea mays dehydrin-1dhn, expressed in the nucleocytoplasm; pTag-WCOR410-RFP, Tricum aestivum cold acclimation protein WCOR410,

32 binding to cellular membranes, and pTag-LEA-BFP, Artemia franciscana LEA protein 33 group 3 that targets the mitochondria. Somatic cells transfected with three LEA proteins 34 were subjected to desiccation under controlled conditions, followed by rehydration, 35 viability assessment and membrane/mitochondria functional tests were performed. Results shown that LEA protect cells from desiccation injury. Cells expressed all LEA proteins shown very high percentage of viable cells (58\%) after four hour of desiccation compare to un-transfected cells (1\% cell alive). Plasmalemma, cytoskeleton and mitochondria appeared unaffected in LEA-expressing cells, confirming their protective action during the entire desiccation and rehydration process. Here, we show that natural xeroprotectants (LEA proteins) transiently expressed in somatic cells confer them desiccation tolerance.

42 


\section{Introduction}

Water is essential for life (Hand et al., 2007; Menze et al., 2009) yet many organisms are able to survive almost completely dehydrated ( $>99 \%$ of their body water is removed) (Crow et al., 1992). In nature, this phenomenon is known as "anhydrobiosis", and is conserved across vegetal and animal phyla (Hincha et al., 1992). Anhydrobiosis allows seeds and small invertebrates to survive long time spans (decades/centuries) in the absence of water, thanks to the induced synthesis of sugars and various protein classes that can be collectively defined as "xero-protectants" (Loi et al., 2013). Among them, Late Embryogenesis Abundant proteins (LEAp) are the best characterized and perhaps the most interesting (Marunde et al., 2013). LEA proteins were first discovered in cotton seeds more than 30 years ago (Dure et al., 1981) and were later also found in seeds and vegetative tissues of several other plants (Shih et al., 2008). A relatively recent survey, probably not updated, contains 769 LEAp entries from 196 organisms (Hunault and Jaspard, 2009). LEA proteins are highly hydrophilic and acquire random coils conformation in aqueous solution, property that has assigned them the definition of "intrinsically disordered" proteins (McCubbin et al., 1985). It is only during de-hydration that LEA proteins acquire their final conformations, primarily $\alpha$ helices, $\beta$ sheets, and hairpin loops, and by doing so they bind to specific cellular/enzymatic substrates to be protected. The mechanism of "xero-protection" is not fully understood. Also lacking are data on the substrate binding mechanism. Data gained in model organisms have indicated that LEAp stabilisation occurs via several pathways: chaperon-like activity, protection of cell membranes, stabilisation of vitrified sugar glasses by increasing glass transition temperature $(\mathrm{Tg})$, sequestration of divalent ions, and synergic interaction with other xero-protectants, such as trehalose (Li et al., 2012). The expression of LEAp, as well as the other xero-protectants, is triggered in anhydrobiotic organisms once water stress is sensed, leading to a progressive accumulation in various cellular compartments, such as mitochondria (Hand et al., 2011; Moore and 
Hand, 2016), nucleus (Wu et al., 2013) cytosol (hand et al., 2011), membranes (Tolleter et al., 2010), and endoplasmic reticulum. Clearly, several LEAp are required, along with other xero-protectants, to confer full protection from water stress. Therefore, if the strategy is to exploit LEAp for the induction of reversible drying in mammalian cells, it is necessary to know their specific targets and the mechanism of action. While the mechanism of action might be inferred from sequence analysis of the LEAp by bioinformatics, in vivo transfection assays of cells with the different LEAp are required to confirm their protective action, but also to exclude negative effects on cell homeostasis. After all, they are mainly vegetal proteins. An index paper published by Li's group was the first to investigate the effects of LEAp expressed in mammalian cells subjected to rapid dehydration (Li et al., 2012). In that work, hepatoma cell line was stably transfected with a tetracycline (Tet)-inducible expression system coding for two LEAp naturally expressed in embryos of the brine shrimp Artemia franciscana, AfrLEA2 and AfrLEA3m, plus a trehalose transporter 1 (TRET1) (Li et al., 2012). The results showed that LEAp, one expressed in the cytoplasm, AfrLEA2, and the other AfrLEA3m in the mitochondria, together with trehalose, effectively protected the cells from the desiccation stress.

Our work extends Li's findings. In addition to the LEAp AfrLEA3m, that targets the mitochondria, we have transfected primary cultures of fibroblasts with two additional LEA proteins: pTag-RAB17-GFP-N, Zea mays dehydrin-1dhn, expressed in the nucleocytoplasm; and pTag- WCOR410-RFP, Tricum aestivum cold acclimation protein WCOR410, that binds to the membranes. Our preliminary work on lyophilized,

100 unprotected, somatic cells directly processed for scanning and electron microscopy showed massive damage at the membrane level [(Matzukawa, personal communication and (Iuso et al., 2012)]. Here reported results show that the individual LEAp protected the cells from 103 desiccation with strongest effect when all three LEA were expressed adding new data on 104 the induction of controlled drying in mammalian cells. 


\section{Results}

106 Subcellular localization of pTag-RAB17-GFP, pTag-WCOR410-RFP, and pTag-

\section{LEA-BFP}

108 Sheep fibroblasts were transiently transfected with pTag-RAB17-GFP, pTag-WCOR410-

109 RFP, pTag-LEA-BFP with efficiency as follow: 44\%, 26\% and 24\%, respectively. pTag-

110 RAB17-GFP was localised to the cytoplasm and nucleus (Fig. 1A-C), pTag-WCOR410-

111 RFP protein was observed only in the cytosol/membranes (Fig. 1G-S) and by localization

112 with membrane dye clearly shown proper localization (Fig. 1M - S), while pTag-LEA-

113 BFP was detected in the mitochondria (Fig.1T - Z). Fig. 1W-Z shows that pTag-LEA-BFP

114 is targeted to the mitochondrial network as it co-localised with MitoTracker green (Fig.

115 1X, Z). Additionally, to confirm the proper localization of the LEA proteins, sheep

116 fibroblasts were transfected with empty vectors EV-GFP, EV-RFP and EV-BFP as a

117 control. Results showed that GFP, RFP and BFP alone had spread distribution throughout

118 the cells (Fig. 1D-F: GFP; Fig. 1J-L: RFP; Fig. 1U: BFP). Expression of fusion LEA

119 proteins in sheep fibroblast was also confirmed by immunoblotting analysis (Fig. 1Z').

120 Moreover, to our knowledge for the first time, we were able to express all tree LEA

121 proteins (pTag-WCOR410-RFP (Fig. 2A); pTag-RAB17-GFP (Fig. 2B); pTag-LEA-BFP

122 (Fig. 2C)) in the same somatic cell, although with low efficiency (11\%). Additionally, in

123 the MIX conditions we have also found cells that expressed only two LEA protein (12\%)

124 as well as cells with single LEAp: pTag-RAB17-GFP (21\%), pTag-WCOR410-RFP (29

$125 \%)$, or pTag-LEA-BFP (14\%).

126

127 Enhanced resistance to desiccation stress

128 Sheep fibroblast transfected with LEA proteins, as well as not transfected controls (CTR),

129 were air dried at $16^{\circ} \mathrm{C}$ for up to $4 \mathrm{~h}$. Every $60 \mathrm{~min}$, cells viability was assessed on sub-

130 samples using trypan blue exclusion. The results showed that $1 \mathrm{~h}$ of air drying did not affect 
131 viability of sheep fibroblasts (Fig. 3A), with more than $80 \%$ of cells expressing any of the

132 LEAp singularly or together and $60 \%$ of CTR maintaining viability (Fig. 3A). One hour

133 later small negative effect of desiccation on cells viability start to be observed but any

134 statistic differences between LEAs and CTR groups were observed. Drastic differences

135 were observed $1 \mathrm{~h}$ later ( $3 \mathrm{~h}$ after initiation of desiccation). The numbers of viable cells

136 expressing a single LEA protein were significantly higher than control group (RAB-17:

137 16\%, WCORB410: 13\%, LEA3: 12\%, CTR: 2\%). By 4h after desiccation begun, only few

138 sheep fibroblast in the CTR were still alive (less than $1 \%$ ) while LEA proteins were able

139 to protect the somatic cells, as indicated by their viability (Rab17: 8\%, WCOR410: 5\%,

140 LEA3: 2.3\%) (Fig. 3A), with the stronger effect observed when all three LEA proteins

141 were co-transfected together (LEA-MIX). In this group, 40\% of LEA-MIX transfected

142 cells were still alive after $3 \mathrm{~h}$ of air drying, compared to $2 \%$ of the CTR group; $23 \%$ of MIX

143 cells were viable after $4 \mathrm{~h}$ of drying while in the control group viability dropped to under

$1441 \%$ (Fig. 3A).

145 To verify viability every hour, we were forced to remove the cells from the drying

146 chamber, and by doing so we exposed them to uncontrolled variation in both humidity and

147 temperature. This could have affected the late time points. For this reason, we decided to

148 carry on the viability tests at two time points: $1 \mathrm{~h}$ and $4 \mathrm{hs}$ post desiccation. Accordingly,

149 stronger difference in cells viability was observed when cells were not exposed to

150 condition changes. Indeed, after $4 \mathrm{~h}$ of desiccation, statistically significant difference

151 between cells expressing single as well as all three LEA together (MIX) and the control

152 group were observed (RAB17: 40\%, WCOR410: 34\%, LEA: 37\%, MIX: 58\%, CTR: 2\%)

153 (Fig. 3B).

154

155 LEA proteins preserve proliferation capacity following desiccation 
156 Transfection of a single LEA protein resulted in a higher number of cells attached to

157 culture dishes (pTag-RAB17-GFP: 55 cells/field; pTag-WCOR410-RFP 62 cells/field;

158 pTag-LEA-BFP: 37 cells/field; LEA-MIX: 86 cells/field) than in cells desiccated without

159 LEA, CTR-D (non- transfected and desiccated cells) (25 cells/field), (Fig.4B). Proliferation

160 rate was $32 \%$ with pTag-RAB17-GFP; $31 \%$ with pTag-WCOR410-RFP; and $25 \%$ with

161 pTag-LEA-BFP (Fig. 4A). In the LEA-MIX group, cell proliferation rate was at levels

162 comparable to the non-desiccated controls (CTR) (48\% vs 51\%, respectively).

163

164 Cellular integrity of mammalian cells after desiccation

165 Somatic cells transfected with LEA proteins and controls (non- transfected and desiccated

166 - CTR-D), were cultured for an additional 24h after desiccation and rehydration. Non-

167 transfected and non-desiccated cells were used as a positive control (CTR). Results showed

168 that LEA proteins protected cellular organelles from desiccation injury (Fig. 5). Cells

169 expressing pTag-WCOR410-RFP (Fig. 5A-H) and pTag-RAB17-GFP (Fig. 5I-R) did not

170 show any post-desiccation damages of the cytoskeleton as shown by F-actin staining (Fig.

$1715 \mathrm{~A}, \mathrm{O})$. Normal actin filaments, spanning the entire cells, were observed in sheep

172 fibroblast expressing these LEA proteins, compared to the CTR group (Fig. 5T). CTR-D

173 cells were less organised and showed fragmented cytoskeleton (Fig. 5Y). Importantly,

174 LEA proteins, and particularly pTag-LEA-BFP, protected the mitochondria in transfected

175 cells (Fig. 6A-D), where high numbers of active mitochondria localized in perinuclear

176 position (Fig. 6A), were observed, similar to the CTR group (Fig. 6E-H). On the other

177 hand, mitochondrial activity was very poor in CTR-D group (Fig. 6I-L) where the

178 organelles were localized peripherally, displaying high fragmentation, and were much less

179 metabolically (Fig. 6I).

180 


\section{Discussion}

183 The natural capacity of simple organisms to survive in a dehydrated state has long been

184 exploited by humankind, with lyophilization as the method of choice for the long-term

185 storage of bacteria and yeast (Fonseca et al., 2015). Some attempts were subsequently

186 conducted to freeze dry non-nucleated mammalian cells, like platelets and red blood cells,

187 with partial success (Crowe et al., 2003). It was the report of the maintenance of nuclear

188 viability in lyophilized spermatozoa (Wakayama and Yanagimachi, 1998) that sparked the

189 interest in dry stabilization of mammalian cells. The low water content and the highly

190 condensed DNA make spermatozoa "easy" to dry, while in contrast other mammalian cells

191 are not tolerant to dehydration and invariably die. No genes related to LEA family have

192 been identified in the mammalian genomes sequences so far, beside one short protein

193 conferring mitochondrial protection (Hall et al., 2011). Therefore, the only possible way to

194 confer cell desiccation tolerance is to provide them with suitable xero-protectants.

196 The first studies reporting on the possibility of drying somatic cells were published by Guo

197 et al., (2000) and Eroglu et al., (2000). These authors showed that it was possible to

198 desiccate and store human fibroblasts for up to 5 days while maintaining viability upon re-

199 hydration. Their method made use of the protective effects of trehalose, a disaccharide

200 associated with organisms withstanding desiccation (Leslie et al., 1995; Welsh and

201 Herbert, 1999). Trehalose was produced in cells previously infected with an adenoviral

202 vector expressing the trehalose biosynthetic genes, ots A and otsB, followed by air-drying 203 and storage at room temperature.

204 Here we followed this general strategy, but LEA proteins were used as xero-protectants.

205 Our work builds on a recent paper where desiccation tolerance was induced in hepatoma

206 cell line expressing Tet-inducible expression system coding for two LEA proteins of the

207 brine shrimp Artemia franciscana, AfrLEA2 and AfrLEA3m, and a trehalose transporter 1 
208 (TRET1). Of the two LEA proteins, AfrLEA2 accumulated in the cytoplasm, and 209 AfrLEA3m selectively targeted the mitochondria. Here we maintained the latter LEA 210 protein, but we selected two different additional ones. In our previous work on 211 lyophilization and nuclear transfer of lyophilized cells, we found high level of DNA 212 damage in the resulting pronuclei (Iuso et al., 2012). Therefore, we elected to use pTag213 RAB17-GFP-N, Zea mays dehydrin-1dhn, that is expressed not only in the cytoplasm as 214 AfrLEA2 does, but also in the nucleoplasm, to protect the DNA as well. The third LEA 215 protein that we utilized was pTag-WCOR410-RFP Tricum aestivum cold acclimation 216 protein WCOR410, that binds specifically to the membranes. This latest LEA proteins was 217 included following our observation of a massive membrane damage in lyophilized 218 fibroblasts processed for scanning electron microscopy (SEM) without re-hydration 219 (Matsukawa, unpublished) and for transmission electron microscopy (TEM) after re220 hydration (Iuso et al., 2012).

222 The proportion of fibroblasts expressing single LEA proteins was around $30 \%$, with no 223 significant differences between the three vectors. Transfection efficiency of all three LEA expression vectors was much lower, about $11 \%$. As a result, the data on cell survival were underestimated because desiccated samples contained both expressing and non-expressing cells. Transgenic cell lines constitutively expressing xero-protectants, as accomplished in Li and co-workers (Li et al., 2012), provide indeed more precise and objective findings, but 228 such approach cannot be an option for practical use.

229 The subcellular localization of all three LEA proteins matched the expectations (Fig. 1 and 230 2), confirming an earlier report (Li et al., 2012). No adverse effects on cell viability were 231 observed in the LEAp expressing cells.

232 The LEA proteins exerted protection against water deprivation, with no major differences 233 between them. LEA pTag-RAB17 expression appeared to be more beneficial over the 
234 other two, probably owing to its ubiquitous expression in all cell compartments (Fig. 3 and

235 4), and also because of its higher transfection efficiency compared to the other two [(44\%

236 vs 26\% (WCOR410-RFP) and 24\% (LEA3-BFP)]. Clearly, cells expressing all three LEA

237 proteins showed the best survival rate, particularly in the experiment with only two time

238 point controls (Fig. 3B). Expression rate of all LEA proteins in the same cells was low

239 (11\%) but presence of other LEAp combinations (single LEAp expression, and

240 combinations of two LEAp in the same cell) increased the protective effects in the MIX

241 group.

242 The proliferation assays after the water stress further demonstrated the beneficial effects of

243 LEA proteins, basically in the cell functions explored - mitochondrial function and

244 distribution, and F-actin (cytoskeleton). Again, no major differences between cells

245 expressing individual LEA protein were detected (Fig. 5), while those expressing the three

246 LEA proteins showed remarkable growth performances, comparable to control, unstressed

247 cells (CTR) (Fig. 4A). Viability was further supported by the normal number and

248 distribution of the mitochondria in growing cells, as well as the normal organization of

249 polarized F-actin across the cells (Fig. 5). In contrary, unprotected cells displayed

250 abnormal mitochondrial distribution (Fig. 6I) and disordered F-actin scattering across the

251 cells (Fig. 5Y), suggesting that time is needed to recover from desiccation damages.

252

253 As learned from anhydrobiotic models, desiccation tolerance is conferred through a

254 combined action of several xero-protectants, including LEA proteins. Our work using a

255 peculiar combination of three LEAp expressing plasmid vectors transferred into sheep

256 fibroblasts, reconfirmed their predicted localizations (Fig. 1), and convincingly

257 demonstrated their protective effects during dehydration, the recovery on rehydration and

258 continued growth following in vitro culture. Our data ameliorate our knowledge on the 
induction of reversible drying in mammalian nucleated cells for their long-term

260 stabilization in an anhydrous state as an alternative biobanking approach.

Materials and methods

\section{Construction of LEAp plasmids}

264 Coding Sequences (CDS) for the LEA protein were produced by gene synthesis (Dundee

265 Cell Products, USA): RAB17 ( 0.5 Kb; Zea mays dehydrin-1dhn, GenBank 266 NM_001111949.1), WCOR410 ( 0.8 Kb; Tricum aestivum cold acclimation protein 267 WCOR410; GenBank L29152.1) and LEA ( 0.9 Kb; Artemia franciscana LEAp group 3; 268 GenBank FJ592175.1). RAB17, WCOR410 and LEA were subcloned into pET-15b 269 (Novagen, Rome, Italy) under T7 promoter. Subsequently, CDS were amplified using 270 AccuPrime Pfx DNA polymerase (ThermoFisher), and inserted using EcoRI/HindIII into 271 the pTag-GFP-N, pTag-RFP-N to obtain pRAB17-GFP and pWCOR410-RFP, and 272 SacI/PstI for the pTag-BFP-N to obtain pLEA-BFP (all plasmid backbones were from 273 Evrogen, Milano, Italy). Correct clones were confirmed by Sanger sequencing using ABI 274 PRISM 3100 (Applied Biosystem).

\section{Transfection}

277 Sheep adult fibroblast (SAF) were derived from ear biopsy of three female Sarda breed 278 sheep (2 years old). Animal work (skin biopsy) has been approved by the Italian Ministry 279 of Health, upon the presentation of the research description prepared by the ethics 280 committee of the Istituto Zooprofilattico Sperimentale di Teramo (Prot. 944F0.1 del $28104 / 11 / 2016$ ). The number of the authorization granted by the Italian Ministry of Health is $282 \mathrm{n}^{\circ}$ 200/2017-PR. We confirm that all methods were performed in accordance with the 283 relevant guidelines and regulations. 
SAFs (between second and eighth passage) were cultured in DMEM (GIBCO) containing

$2 \mathrm{nM}$ glutamine, $3.7 \mathrm{~g} / \mathrm{L} \mathrm{NaHCO} 3$ and $0.5 \%$ gentamicin supplemented with $10 \%$ Fetal

287 Bovine Serum (FBS).

288 Transfection of sheep adult fibroblasts was adapted from Czernik and colleagues (Czernik 289 et al., 2016) with small modifications. Approximately $10^{6}$ cells were plated in $3,56 \mathrm{~cm}$ 290 dishes and cultured in Minimal Essential Medium (MEM) $+10 \%$ foetal bovina serum 291 (FBS) (Gibco, Milan, Italy) for 24h. After 24h cells were transfected using Lipofectamine 2922000 kit (Invitrogen, Milan Italy), according to the manufacturer's protocol, using $3 \mu \mathrm{g}$ of 293 pTag-RAB17-GFP-N, pTag-WCOR410-RFP-N, pTag-LEA-BFP, -individually or in 294 combination (by $2 \mu \mathrm{g}$ of each) (called MIX). Additionally, empty vectors: pTags-GFP-N, 295 pTags-RFP-N, pTags-BFP-N were used as a control (called EV-GFP, EV-RFP, EV-BFP, 296 respectively). After transfection cells were incubated in a humidified atmosphere 5\% $297 \mathrm{CO}_{2} / 95 \%$ air at $37^{\circ} \mathrm{C}$. All experiments were done $24 \mathrm{~h}$ post-transfection. Efficiency of the 298 transfection of all experiments were normalised normalized by using an internal control,

\section{Localization of LEA proteins in somatic cells}

301 Sheep fibroblasts transfected with pTag-RAB17-GFP, pTag-WCOR410-RFP, pTag-LEA-

BFP individually or in combination, as well as empty vector controls, EV-GFP, EV-RFP,

EV-BFP, were fixed with $4 \%$ paraformaldehyde (PFA) for $20 \mathrm{~min}$ at room temperature

304 (RT). After subsequent wash with PBS, cells were counterstained for $10 \mathrm{~min}$ with $5 \mu \mathrm{g} / \mathrm{mL}$

305 Hoechst 33342 (pTag-RAB17-GFP and pTag-WCOR410-RFP cells) or $0.5 \mu \mathrm{g} / \mathrm{mL}$

306 Propidium Iodide (PI) (pTag-LEA-BFP expressed cells). Then, cells were mounted on

307 slides with Fluoromount ${ }^{\mathrm{TM}}$ aqueous mounting medium (Sigma, Milan, Italy) and

308 localization of the proteins was analysed with using Nikon Ar1 laser confocal scanning

309 microscope (Nikon Eclipse Ti-E) equipped with the NIS- Element 4.40 software. 


\section{Western Blot}

312 Proteins were isolated from sheep fibroblasts transfected with pTag-RAB17-GFP, pTag-

313 WCOR410-RFP, pTag-LEA-BFP, EV-GFP, EV-RFP, EV-BFP and from the mock

314 transfection control, by incubation overnight with lysis buffer I (20 mM Tris, $150 \mathrm{Mm}$

$\left.315 \mathrm{NaCl}_{2}, 1 \% \mathrm{NP}-40\right)$ at $4^{\circ} \mathrm{C}$. Then protein extracts were re-suspended at 1:1 ratio in lysis

316 buffer II (20 Mm Tris, $150 \mathrm{Mm} \mathrm{NaCl}_{2}$ ). Protein concentration was assessed using the BCA

317 Protein Assay Kit (Thermo-Fisher, Milan, Italy) according to the manufacture protocol.

318 For each sample $50 \mu \mathrm{g}$ of protein were incubated at $95^{\circ} \mathrm{C}$ for $10 \mathrm{~min}$ and then loaded into a

319 gradient (4-15\%) western blot gel (mini-protein TGX gel, Bio-Rad, Milan, Italy). Proteins

320 were transferred onto a $0.45 \mu \mathrm{m}$ nitrocellulose membrane (Bio-Rad, Milan, Italy) at $4{ }^{\circ} \mathrm{C}$

321 for $2 \mathrm{~h}$ at $200 \mathrm{~mA}$. After transfer, the nitrocellulose membrane was blocked with $5 \%$ non-

322 fat dry milk in $0.1 \%$ Tween-20 PBS (PBST) for $1 \mathrm{~h}$ at RT. Membranes were incubated

323 overnight with rabbit anti-tagRFP (which recognise also tagBFP) or rabbit anti-

$324 \operatorname{tag}(\mathrm{CGY})$ FP primary antibodies (both from Evrogen, Milan, Italy) at 1:5000 in PBST with

$3250.5 \%$ non-fat dry milk. Then, membranes were washed three times for 15 min with PBST

326 and incubated with the secondary antibody donkey anti-rabbit- IgG-HRP (sc-2317, Santa

327 Cruz Biotechnology, USA) at 1:10000 for $1 \mathrm{~h}$ at RT. Final detection was performed using

328 enhanced chemiluminescence (ECL) Western Blotting Substrate (Amersham-Pharmacia,

329 Piscataway, NJ, USA) and image acquisition that was carried out using the ChemiDoc

330 System (Bio-Rad, Milan, Italy). Western blot analysis were repeated 4 times.

332 Desiccation of sheep fibroblasts expressing LEA proteins, cell viability, and residual

333 water

334 Sheep fibroblasts transfected with pTag-RAB17-GFP, pTag-WCOR410-RFP, pTag-LEA-

335 BFP, individually or in combination, were detached with Trypsin-EDTA $(0.25 \%)$ and 336 pelleted by spinning them for $5 \mathrm{~min}$ at $1200 \mathrm{rpm}$ (Eppendorf Centrifuge 5804). Cells were 
337 then re-suspended to $10^{5}$ cells $/ \mathrm{mL}$ in desiccation medium $(50 \mathrm{mM}$ Hepes and $500 \mathrm{mM}$

338 trehalose in PBS) and drops of $10 \mu \mathrm{L}$ were placed on a plastic, cover dish. Cells were then

339 air-dried at $16^{\circ} \mathrm{C}$ for 1,23 and $4 \mathrm{~h}$. After desiccation, cells were rehydrated by adding $50 \mu \mathrm{L}$

340 of the same desiccation medium and incubated for $5 \mathrm{~min}$ at RT. Viability and number of

341 alive cells were evaluated using Trypan Blue staining. Un-transfected cells were used as a

342 control (CTR). Residual water was assessed by weighing first the empty cover dish, then

343 weighing the samples before drying and again after drying. This gave us a value of residual

344 water per dry weight. Desiccation and residual water assessment was done 15 times.

346 Cell proliferation assay

347 After desiccation, the fibroblasts were transferred into culture medium (MEM $+10 \%$ FBS)

348 and cultured in a humidified atmosphere $\left(5 \% \mathrm{CO}_{2} / 95 \%\right.$ air at $\left.37^{\circ} \mathrm{C}\right)$ for $24 \mathrm{~h}$. Cell

349 proliferation was assessed by indirect immunocytochemistry detection of 5-bromo-2'-

350 deoxyuridine (BrdU), a thymidine analogy incorporated during the S-phase of the cell

351 cycle. Briefly, cells were cultured with $100 \mu \mathrm{M}$ BrdU for 6 hours before the end of culture,

352 fixed in cold $100 \%$ methanol for $20 \mathrm{~min}$, and permeabilised with $0.1 \%$ Triton-X-100 in

353 PBS for $15 \mathrm{~min}$ at RT. Next, cells were treated with $4 \mathrm{~N} \mathrm{HCl}$ at RT for $30 \mathrm{~min}$ and

354 incubated with mouse anti-BrdU at 1:100 (B2531, Sigma, Milan, Italy) in blocking

355 solution $\left(0.1 \%\right.$ BSA in PBS) over-night at $4{ }^{\circ} \mathrm{C}$, overnight. Cells were then incubated with

356 rabbit anti-mouse IgG-FITC polyclonal antibody at 1:500 (F9137, Sigma, Milan, Italy) in

357 blocking solution at RT for $2 \mathrm{~h}$ and counterstained with $0.5 \mu \mathrm{g} / \mathrm{mL}$ PI at RT for $5 \mathrm{~min}$.

358 Between passages, cells were washed twice with PBS at RT for 5 min. Proliferation assay

359 were repeated 5 times and at every repeat 10 different field/sample were photographed.

360 The number of proliferative cells (expressed BrdU) vs. total cells number were

361 automatically counted using Nikon Ar1 laser confocal scanning microscope (Nikon Eclipse

362 Ti-E) equipped with the NIS- Element 4.40 software. 


\section{Mitochondrial and cytoskeleton staining}

364 After desiccation, the fibroblasts were transferred into the culture medium (MEM $+10 \%$

365 FBS $)$ and cultured in a humidified atmosphere $\left(5 \% \mathrm{CO}_{2} / 95 \%\right.$ air at $\left.37^{\circ} \mathrm{C}\right)$ for $24 \mathrm{~h}$. The

366 cells were then incubated with $1 \mu \mathrm{M}$ of Mitotracker green FM (Invitrogen, Molecular

367 Probes, Milan, Italy) for mitochondrial staining or $1 \mu \mathrm{M}$ of Phalloidin green

368 (Thermofisher, Milan, Italy) for F-actin (the cytoskeleton) staining, both in serum-free

369 MEM for $30 \mathrm{~min}$ at $38.5^{\circ} \mathrm{C}$. Then, cells were washed twice with PBS, fixed with $4 \%$

370 paraformaldehyde and counterstained with $5 \mu \mathrm{g} / \mathrm{mL}$ of Hoechst 33342. All slides were

371 examined by confocal microscopy (Nikon Eclipse Ti-E) using NIS-Elements Confocal

372 software (Nikon).

\section{Statistical analysis}

374 One-way ANOVA and Fisher exact test were used to compare live cells at different

375 durations of desiccation. Data reported in this paper are the mean $( \pm$ SEM) for each group.

376 The level of significance was set at $P<0.05$. Statistical analyses were performed using

377 GraphPad Prism for Windows (Version 6.01, GraphPad Software, Inc, CA, USA).

\section{Acknowledgements}

380 The project has received funding from the European Union's Horizon 2020 Research and

381 Innovation Programme under the Marie Skłodowska-Curie grant agreement No 734434

382 (DRYNET) and by Narodowe Centrum Nauki (NCN) GA 2016/21/D/NZ3/02610 to MCz.

\section{Author Contributions}

385 M.C, A.F conceived and designed the research; M.C, A.F, FP.L performed the experiments;

386 M.C, A.F, FP. L, L.V analyzed the data; AP.S and PP edit the paper M.C, A.F and P.L wrote

387 the paper. All the authors discussed the results and contributed to the writing edited and 388 reviewed the manuscript. 


\section{Declaration of Interests}

390 The authors declare no competing interests.

391

392

393

394

395

396

397

398

399

400

401

402

403

404

405

406

407

408

409

410

411

412

413

414

\section{References}

1. Hand, S.C., Jones, D., Menze, M.A., Witt, T.L. (2007). Life without water: Expression of plant LEA genes by an anhydrobiotic arthropod. J Exp Zool A Ecol Genet Physiol 307, 62-66.

2. Menze, M.A., Boswell, L., Toner, M., Hand, S.C. (2009). Occurrence of mitochondria-targeted late embryogenesis abundant (LEA) gene in animals increases organelle resistance to water stress. J Biol Chem 284, 10714-10719

3. Crowe, J.H., Hoekstra, F.A., Crowe, L.M. (1992). Anhydrobiosis. Annu. Rev. Physiol 54, 579-99

4. Hincha, D.K., Thalhammer, A. (2012). LEA proteins IDPs with versatile functions in cellular dehydration tolerance. Biochem Soc Trans. 40(5), 1000-3

5. Loi, P., Iuso, D., Czernik, M., Zacchini, F., Ptak, Towards storage of cells and gametes in dry form. G. (2013). Trends Biotechnol 31(12), 688-95

6. Marunde, M.R., Samarajeewa, D.A., Anderson, J., Li, S., Hand, S.C., Menze, M.A. (2013).Improved tolerance to salt and water stress in Drosophila melanogaster cells conferred by late embryogenesis abundant protein. J Insect Physiol. 59, 377-86

7. Dure, L., Greenway, S.C., Galau, G.A. (1981). Developmental biochemistry of cottonseed embryogenesis and germination: changing messenger ribonucleic acid populations as shown by in vitro and in vivo protein synthesis Biochemistry 20, $4162-4168$.

8. Shih, M.D., Hoekstra, F.A., Hsing, Y.I.C. Late embryogenesis abundant proteins. In K. Jean-Claude \& D. Michel (Eds.), Advances in botanical research (2008).

9. Hunault, G., Jaspard, E. (2009). LEA Pdb: a database for the late embryogenesis abundant proteins BMC Genomic s2010, 11:22.1. 
10. McCubbin, W.D., Kay, C.M., Lane, B. (1985). Hydrodynamic and optical properties of the wheat germ Em protein. Can J Biochem Cell Biol 63, 803-811

11. Li, S., Chakraborty, N., Borcar, A., Menze, M.A., Toner, M., Hand, S.C. (2012). Late embryogenesis abundant proteins protect human hepatoma cells during acute $\mathrm{d}$ esiccation. Proc Natl Acad Sci U S A. Dec 18;109(51), 20859-64

12. Hand, S.C., Menze, M.A., Toner, M., Boswell, L., Moore, D. (2011). LEA proteins during water stress: not just for plants anymore. Annu. Rev. Physiol. 73, 115-134.

13. Moore D.S., Hand, S.C. (2016). Cryopreservation of lipid bilayers by LEA proteins from Artemia franciscana and trehalose Cryobiology. 2016 Oct;73(2), 240-7. protein EMB564 within the cell. Bioinformation. 9(6), 276-80.

15. Tolleter, D., Hincha, D.K., Macherel, D. (2010). A mitochondrial late embryogenesis abundant protein stabilizes model membranes in the dry state. Biochim. Biophys. Acta 1798, 1926-1933.

16. Iuso, D., Czernik, M., Di Egidio, F., Sampino, S., Zacchini, F., Bochenek, M., Smorag, Z., Modlinski, J.A., Ptak, G., Loi, P. Genomic Stability of Lyophilized sheep somatic cells before and aftern nuclear transfer. PLos One 8, 1-8 (2012). Methods Mol Biol. 1257, 477-88. (2003). Stabilization of membranes in human platelets freeze-dried with trehalose. Chem Phys Lipids 122(-2), 41-52. oocytes injected with freeze-dried spermatozoa. Nat Biotechnol 16, 639-41. 
20. Hall, B.M., Owens, K.M., Singh, K.K. (2011). Distinct functions of evolutionary conserved MSF1 and late embryogenesis abundant (LEA)-like domains in mitochondria. J Biol Chem. 286 (45), 39141-39152.

21. Guo, N., Puhlev, I., Brown, D.R., Mansbridge, J., Levine, F. (2000). Trehalose expression confers desiccation tolerance on human cells. Nat Biotechnol 18, $168-$ 171.

22. Eroglu, A., Russo, M.J., Bieganski, R., Fowler, A., Cheley, S., Bayley, H., Toner, M. (2000). Intracellular trehalose improves the survival of cryopreserved mammalian cells. Nat Biotechnol 18, 163-167. and sucrose protect both membranes and proteins in intact bacteria during drying. Appl. Environ. Microbiol 61, 3592-3597.

24. Welsh, D.T., Herbert, R.A. (1999). Osmotically induced intracellular trehalose, but not glycine betaine accumulation promotes desiccation tolerance in Escherichia coli. FEMS Microbiol. Lett. 174, 57-63

25. Czernik, M., Iuso, D., Toschi, P., Khochbin, S., Loi, P. (2016). Remodeling somatic nuclei via exogenous expression of protamine 1 to create spermatid-like structures for somatic nuclear transfer. Nat Protoc 11, 2170-2188.

\section{Figure legend}

459 Figure 1. Subcellular localization of individual LEA proteins in sheep fibroblasts. (A460 C) pTag-RAB17-GFP; (D - F) GFP-tag; (G - I) pTag-WCOR410-RFP; (J - L) RFP-tag; 461 (M - S) pTag-WCOR410-RFP and membrane. (A) pTag-RAB17-GFP fusion protein 462 shows cytoplasmic and nuclear localization (green arrows head, A); (B) nucleus stained 463 with Hoechst 33342; (C) merge; (D) EV control expressing only GFP was spread 464 throughout the cells; (E) nuclei stained with Hoechst 33342, (F) merge; (G) pTag- 
WCOR410-RFP fusion protein exhibit membrane localization (red arrow); (H) nuclei

466 stained with Hoechst 33342; (I) merge; (J) RFP alone show spread localization in the cells;

467 (K) nuclei stained with Hoechst 33342 (L) merge; (M) pTag-WCOR410-RFP fusion 468 protein exhibit membrane localization (red arrow); (N) nuclei stained with Hoechst 33342;

469 (O) membrane staning, (P) merge, (R, S) enlarge Fig. 1P; (T) pTag-LEA3-BFP shows 470 mitochondrial localization, compared to (U) BFP alone that is spread throughout the cell; 471 (W - Z) pTag-LEA3-BFP co-localizes with Mitotracker green; (W) expression of pTag472 LEA3-BFP; (X) mitochondria stained with Mitotracker green dye; (Y) nuclei stained with 473 Propidio Iode; $(Z)$ merge; ( $\left.Z^{\prime}\right)$ expression of LEA proteins in sheep fibroblasts was 474 verified by western blot.

475

Figure 2. Subcellular localization of all three LEA proteins in sheep fibroblasts. (A)

GFP fusion protein showed cytoplasmic and nuclear localization and mitochondira stained with Mitotracker green, (C) pTag-LEA-BFP showed mitochondrial localization as well as 480 nucleus stained with Hoechst 33342 (D) merge. Red - RFP; green - GFP and Mitotracker green dye; blue - Hoechst 33342.

482

Figure 3. Enhanced resistance to desiccation stress. (A) Sheep fibroblast transfected with single LEA protein or with all three LEA proteins (here called MIX). Viability of the 485 cells was controlled every hour for up to four hours using Tripan Blue staining; ** means 486 value $\mathrm{P}=0.0052$ CTR vs MIX (3 and 4 hours) (B) Sheep fibroblast transfected with single 487 LEA protein or with MIX. Viability of the cells was controlled at two time points (after $1 \mathrm{~h}$ 488 and 4 h of controlled drying); **means $\mathrm{P}=0.03 \mathrm{CTR}$ vs RAB17 after $1 \mathrm{~h}$ of desiccation; *** 489 means $\mathrm{P}=0.0046 \mathrm{CTR}$ vs MIX after $1 \mathrm{~h}$ and $4 \mathrm{~h}$ of desiccation; ${ }^{*} \mathrm{p}<0,05$ CTR vs single 490 LEAs $4 \mathrm{~h}$ post desiccation. 
492 Figure 4. LEA proteins preserve proliferation capacity following desiccation. (A)

493 Proliferation was measured by counting of cells that had incorporated BrdU in the nuclei;

$494 * * \mathrm{p}<0,005 ; * * * \mathrm{p}<0,0005$; (B) Attached cells count after desiccation, re-hydration and 495 culture for $24 \mathrm{~h}$ in incubator [(transfected with single LEA proteins: pTag-RAB17-GFP, 496 pTag-WCOR410-RFP, pTag-LEA-BFP or all together (MIX)];

498 Figure 5. Integrity of mammalian cells after desiccation. (A-H) sheep fibroblasts 499 expressing pTag-RAB17-GFP; (I-R) sheep fibroblasts expressing pTag-WCOR410-RFP; 500 (S-W) non desiccated control sheep fibroblasts (CTR); (X-Z') non transfected sheep 501 fibroblasts, after desiccation (CTR-D); (A, O, T, Y) cytoskeleton, stained with Phalloidin 502 green; (I, N, S, X) mitochondria, stained with Mitotracker red dye; (E) mitochondria, 503 stained with Mitotracker green dye; (C, G, L, P, U, Z) nucleus, stained with Hoechst 504 33342; (D, H, M, R, W, Z') merge;

Figure 6. pTag-LEA-BFP protects the mitochondria from desiccation damages. (A - 
bioRxiv preprint doi: https://doi.org/10.1101/704809; this version posted July 16, 2019. The copyright holder for this preprint (which was not certified by peer review) is the author/funder, who has granted bioRxiv a license to display the preprint in perpetuity. It is made available under aCC-BY-NC-ND 4.0 International license. 


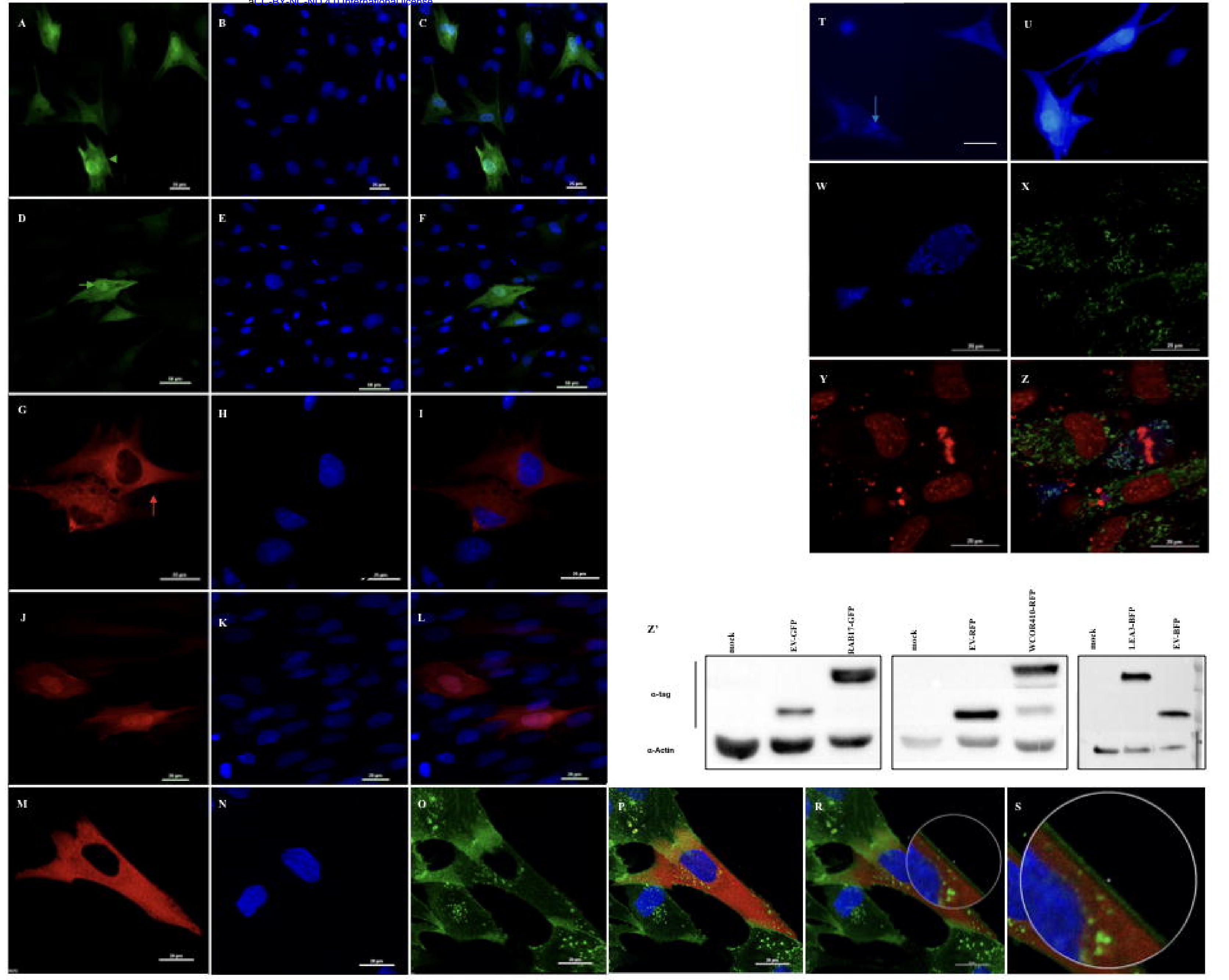


Figure 2.

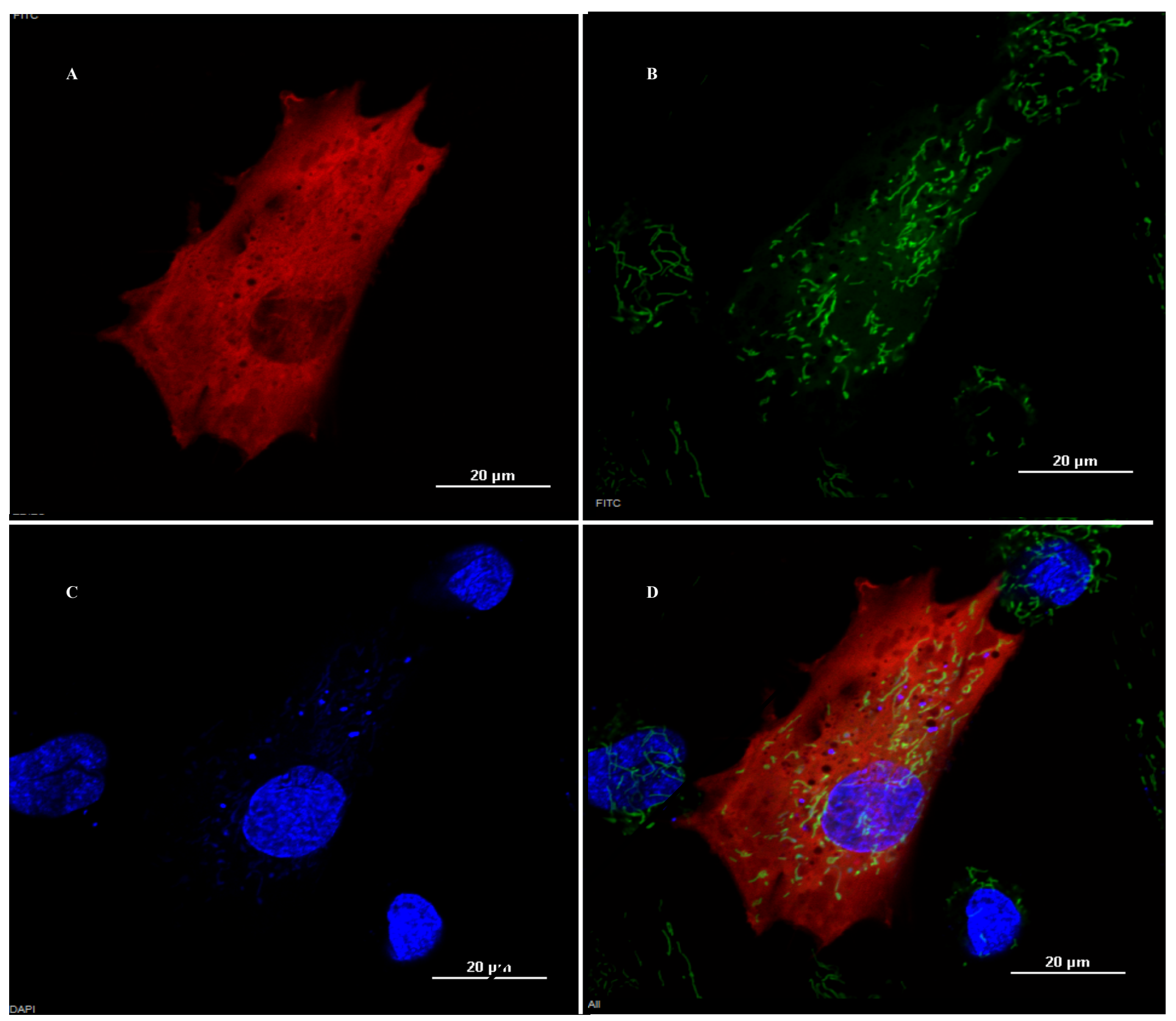




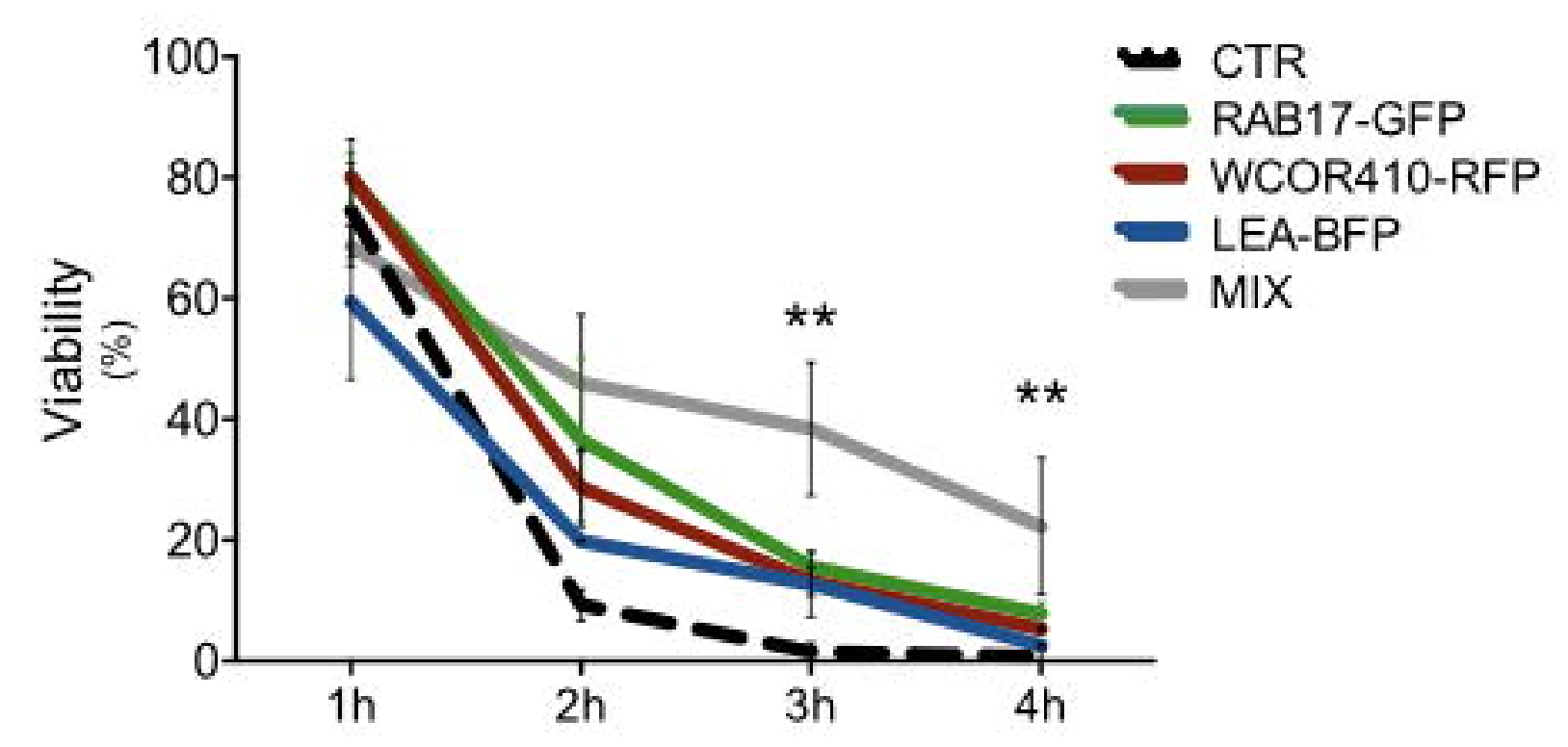

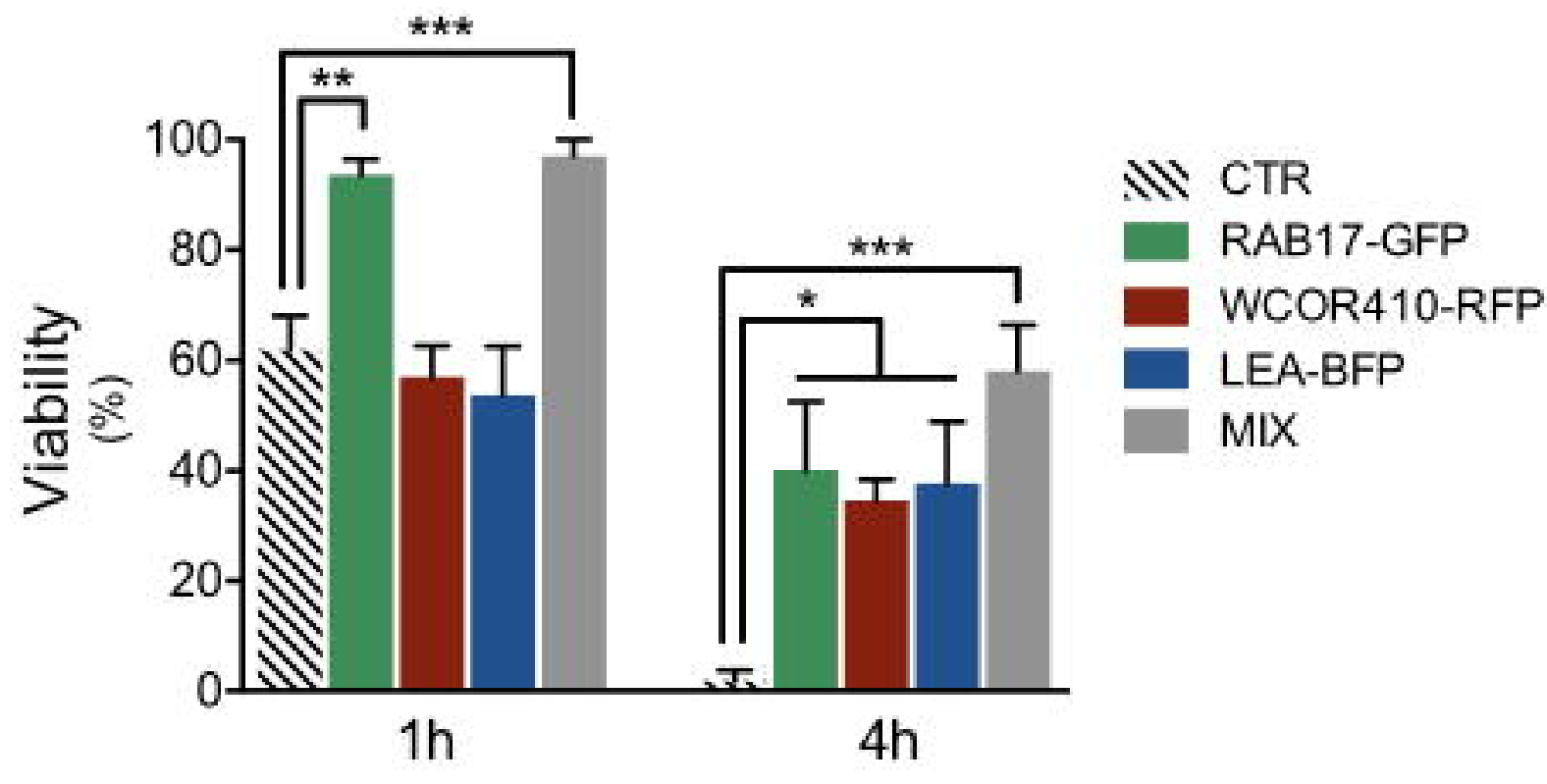




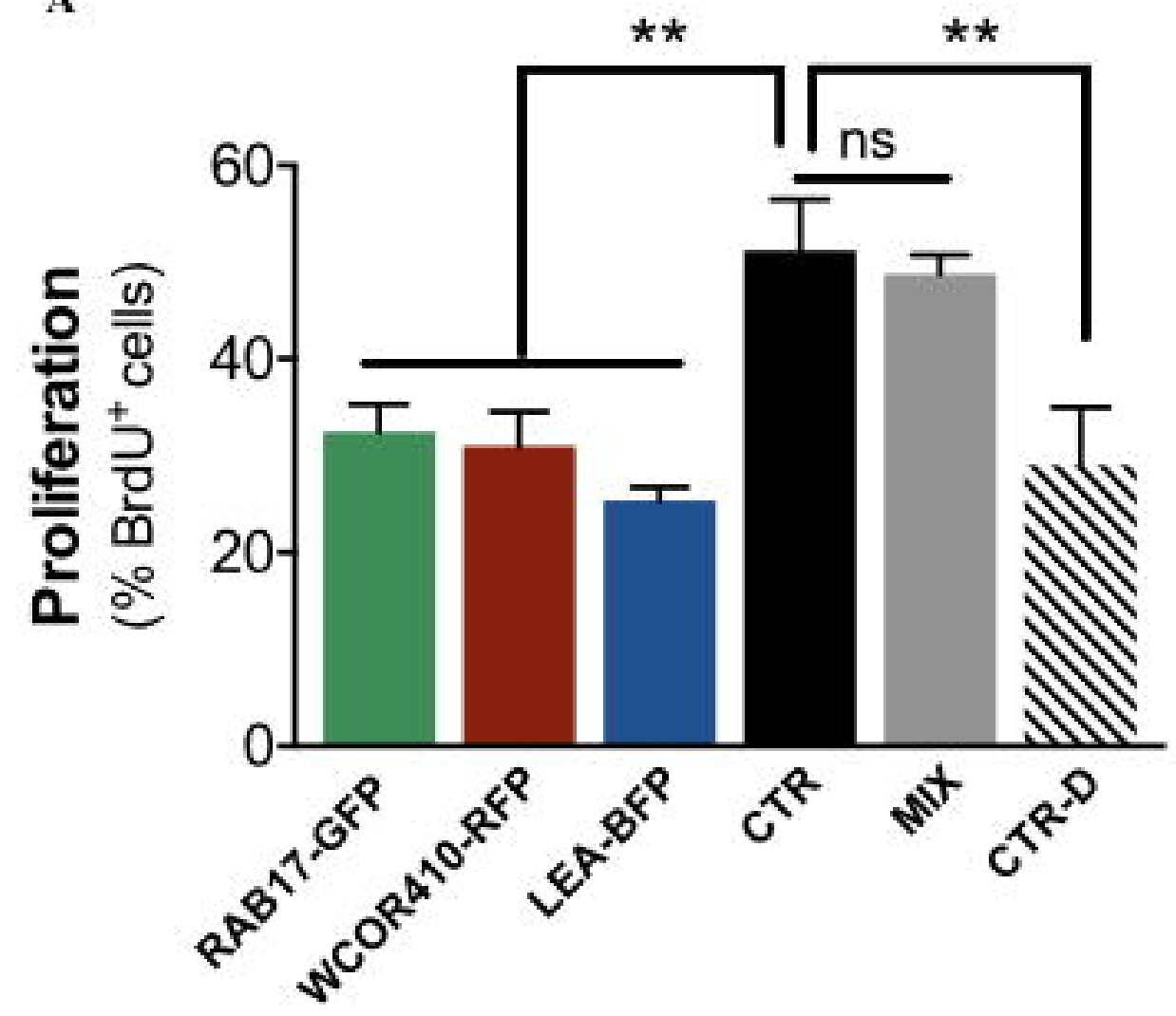

B

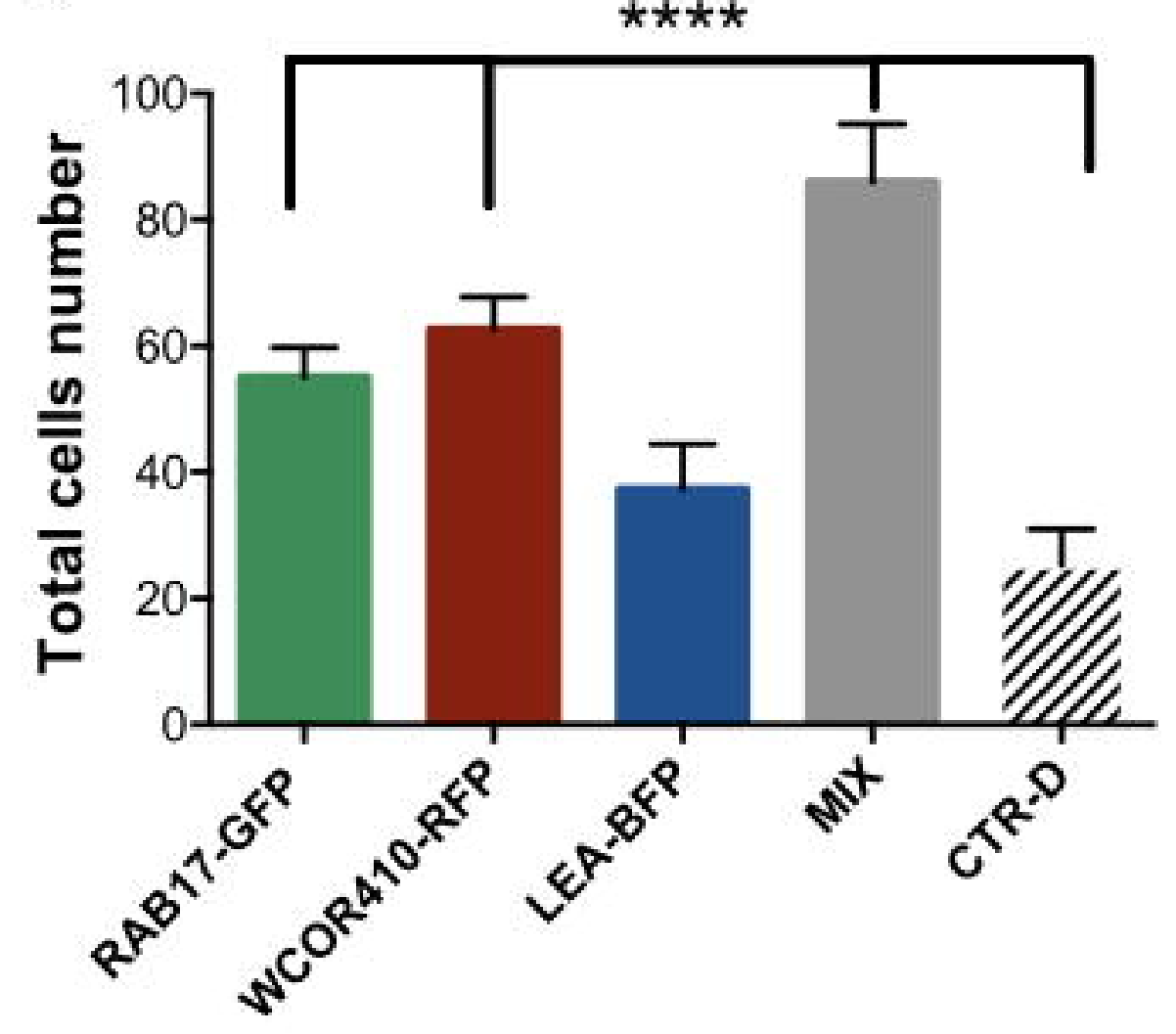




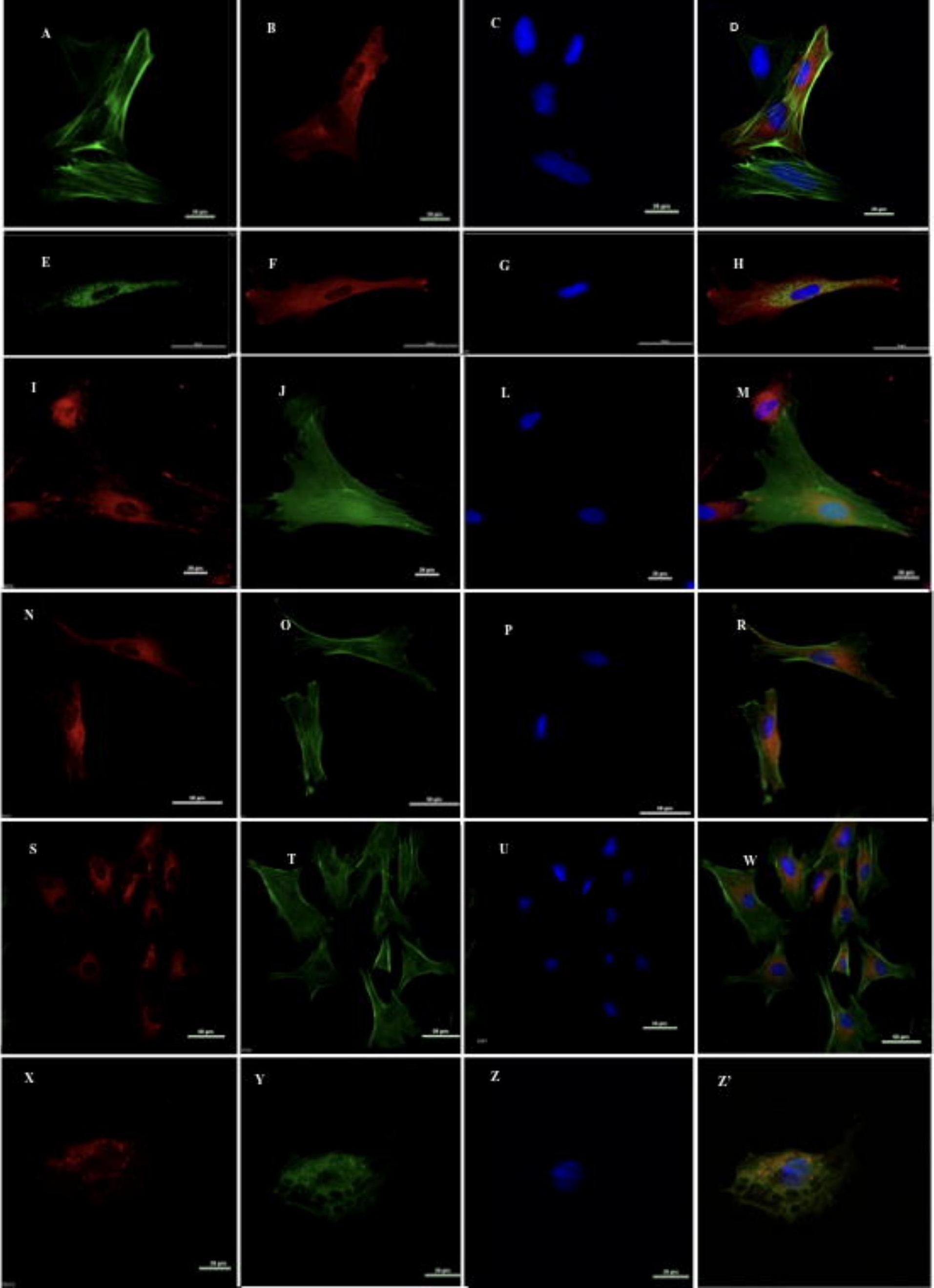




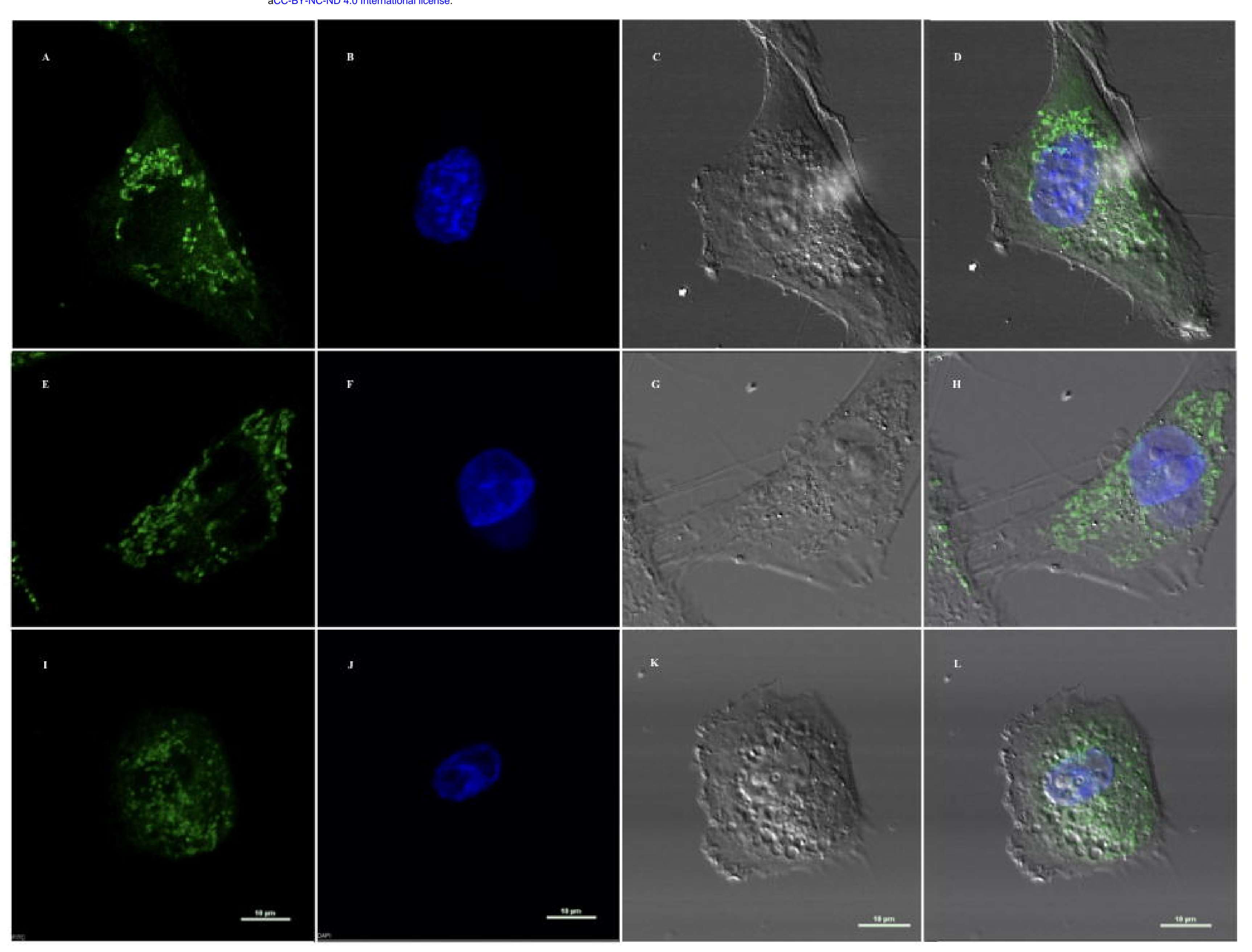

